\title{
Imagen de Hipertrofia Bilateral del Proceso Coronoides Mandibular a Través de la Tomografía Computarizada Cone-Beam
}

\author{
Image of Bilateral Hypertrophy of the Mandibular Coronoid Process Through Cone-Beam \\ Computed Tomography
}

Nilton Alves ${ }^{*} \&$ Naira Figueiredo Deana ${ }^{* *}$

\begin{abstract}
ALVES. N. \& FIGUEIREDO, D. N. Imagen de hipertrofia bilateral del proceso coronoides mandibular a través de la tomografía computarizada cone-beam. Int. J. Odontostomat., 6(2):225-228, 2012.

RESUMEN: La hipertrofia del proceso coronoides mandibular es una patología rara, progresiva y de etiología desconocida, que se caracteriza por el desarrollo exagerado del proceso coronoides determinando aumento en su tamaño, resultando en una progresiva y dolorosa limitación de la apertura bucal. La hipomovilidad mandibular es un síntoma presente en diversas enfermedades y asociarla a una causa específica requiere exámenes clínicos e imagenológicos minuciosos. La tomografía computarizada cone-beam tiene el potencial de ofrecer informaciones complementarias superiores a las radiografías convencionales. Además, elimina superposiciones, ofrece alta resolución de imagen, lo que ayuda no solo el diagnóstico, sino también la planificación del procedimiento quirúrgico. Presentamos un caso de paciente de sexo masculino que acudió a la consulta para evaluación de trismus. No presentaba dolor, solo incomodidad por limitación progresiva de apertura bucal. Mediante el examen físico, solo se observó la limitación de apertura bucal. Aunque los movimientos de lateralidad y protrusión eran posibles, estaban muy limitados. Se realizó examen de tomografía computarizada cone-beam, que evidenció imagen compatible con hipertrofia bilateral del proceso coronoides mandibular.
\end{abstract}

PALABRAS CLAVE: hipertrofia, proceso coronoides mandibular, hipomovilidad mandibular, tomografía computarizada Cone-Beam.

\section{INTRODUCCIÓN}

La dificultad en abrir la boca es un síntoma presente en varias situaciones, tales como trastornos temporomandibulares, anquilosis de la articulación temporomandibular, trismus post-radioterapia, entre otras. La hipomovilidad mandibular es fácilmente detectable durante el examen clínico, sin embargo, asociarla a una causa específica no siempre es senciIlo. La hipertrofia idiopática del proceso coronoides mandibular, que se caracteriza por el desarrollo exagerado del proceso coronoides determinando aumento en su tamaño, está asociada con una progresiva y dolorosa limitación de apertura de boca (Thiegui et al., 2005; Mazzeto \& Hotta, 2007). Suele ser mal diagnosticada, dado que se trata de una condición clínica relativamente rara con evolución generalmente lenta y progresiva (Thiegui et al.). Los movimientos de protrusión y lateralidad pueden quedarse restringidos por el contacto que hace el proceso coronoides con el arco cigomático. Esto se hace más evidente durante la apertura máxima de boca (lqbal et al., 2009). Pueden estar presentes asimetría facial, dolor durante la apertura, además de una masa móvil sobre el arco cigomático (Iqbal et al.; Jamal et al., 2009). La asimetría facial es frecuentemente observada en los casos de hipertrofia unilateral (lqbal et al.).

Para el correcto diagnóstico de una condición clínica con tales características se hace necesario el examen imagenológico. Entre las muchas opciones de examen existentes destacamos la tomografía computarizada cone-beam que además de ofrecer alta resolución en la imagen, permite obtener reconstrucciones de todas las tomas radiográficas convencionales utilizadas en Odontología.

\footnotetext{
* Universidad Estadual Paulista Júlio de Mesquita Filho, UNESP, Araraquara, Brasil.

"Kinesiólogo, Araraquara, Brasil.
} 


\section{REPORTE DEL CASO}

Individuo de sexo masculino, caucásico de 42 años de edad, asiste a la consulta para evaluación de trismus que tenía desde hace largo tiempo. No presentaba dolor, solo incomodidad por limitación progresiva de apertura bucal. La anamnesis no reveló información relevante asociada al problema. Mediante el examen físico, nada se constató, solamente limitación de apertura bucal. Aunque los movimientos de lateralidad y protrusión eran posibles, estaban muy limitados. Se realizó examen de tomografía computarizada cone-beam, que evidenció imagen compatible con hipertrofia bilateral del proceso coronoides mandibular (Figs. 1A y 1B).
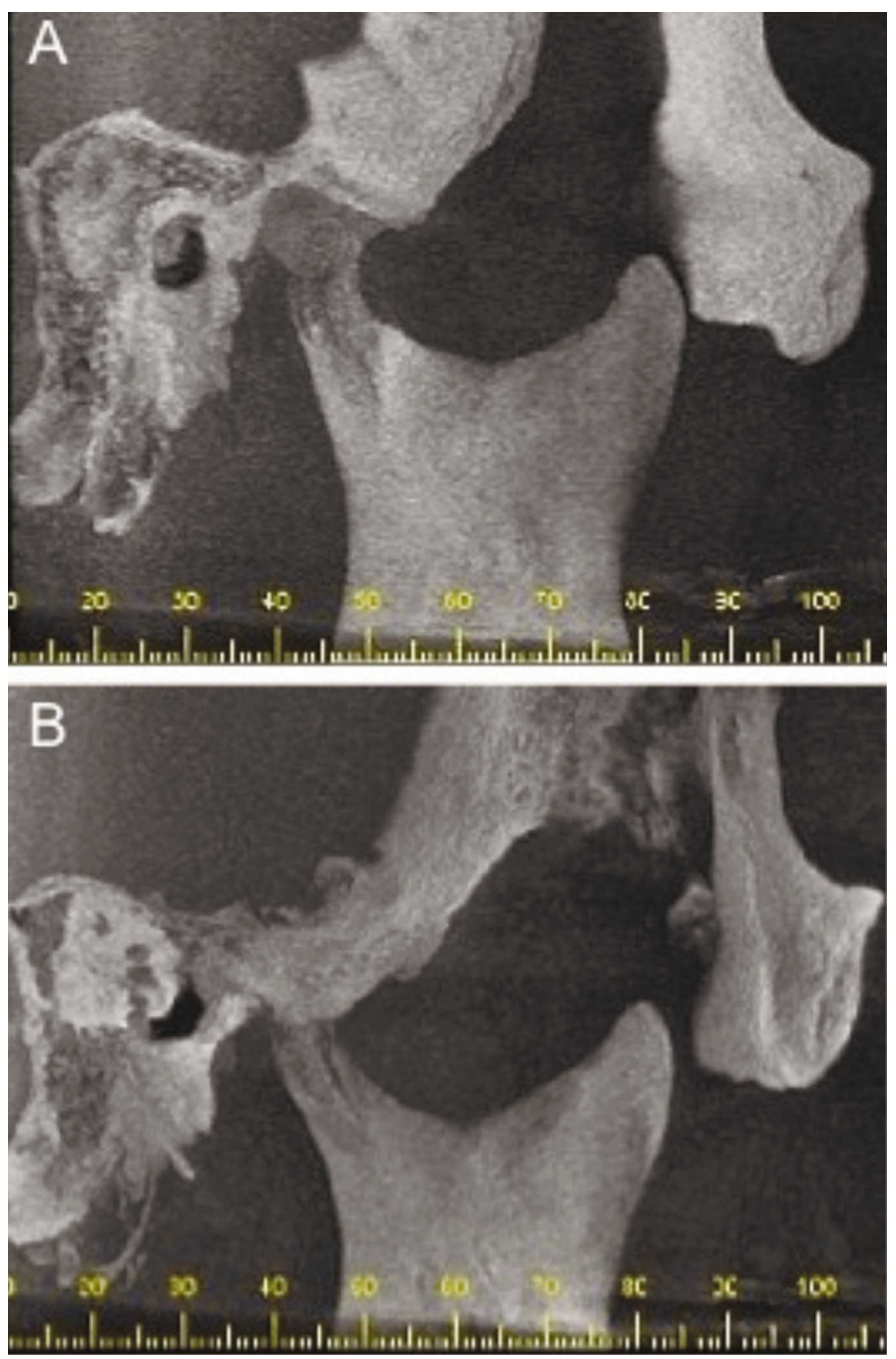

Fig. 1. Imagen de Tomografía Computarizada Cone-Beam compatible con hipertrofia del proceso coronoides mandibular. A. Lado derecho. B. Lado izquierdo. En ambas imágenes el proceso cigomático ha sido removido para facilitar la visualización del proceso coronoides hipertrofiado.

\section{DISCUSIÓN}

La etiología de la hipertrofia del proceso coronoides mandibular es discutida y se han planteado diversas teorías (Thiegui et al.; Iqbal et al.; Jamal et al.). Factores tales como estímulo endocrino, trauma, herencia genética y aumento en la actividad del músculo temporal han sido sugeridos como responsables por este desorden de crecimiento. El proceso coronoides mandibular sirve de inserción para el fascículo superficial del músculo temporal (Alves \& Cândido, 2008, 2012). En relación a la hiperactividad del músculo temporal, distintos autores afirman que se trata de un factor etiológico relevante, ya que la continua acción de los músculos produce un aumento del aporte vascular local produciendo cambios degenerativos y aposición de calcio con consecuente osificación local de tejidos blandos (Kai et al., 1997; Roychoudbury et al., 2002). Aún no existen evidencias que soporten ninguna de esas teorías (Jamal et al.). Actualmente es considerada una enfermedad idiopática, que puede ser unilateral o bilateral.

La hipertrofia bilateral del proceso coronoides afecta a personas de ambos sexos, generalmente hombres, durante el desarrollo del esqueleto facial. En general ocurre en la puberdad y se continua con el paso de los años (Izumi et al., 2005; Wenghoefer et al., 2008).

La hipertrofia del tipo secundario está asociada con alteraciones en la articulación temporomandibular, que suele ser unilateral. En este tipo, los mecanismos de estrés en el tendón del músculo temporal no son compensados por el cóndilo (Pregarz et al., 1998).

El correcto diagnóstico de la hipertrofia del proceso condilar mandibular involucra el examen imagenológico, que incluso también servirá para una adecuada planificación del tratamiento.

En este trabajo presentamos un caso en el cual el paciente presentaba limitación de apertura bucal sin cualquier otra información importante en la anamnesis. Mediante el examen físico se observó que además de la limitación de apertura bucal, también estaban muy limitados los movimientos de lateralidad y protrusión. Delante de tales características clínicas se decidió someter al paciente a un examen imagenológico. 
La tomografía computarizada cone-beam es un método de diagnóstico por imagen que presenta muchas ventajas, principalmente el reducido costo y la menor dosis de radiación sumados a la alta calidad de imagen. A través del programa cone-beam se permite la reconstrucción multiplanar del volumen escaneado (imágenes axiales, coronales, sagitales y oblicuas) e imagen en 3-D. Además se puede generar imágenes bidimensionales réplicas de las radiografías convencionales, como la panorámica o las telerradiografías en norma lateral y frontal (Scarfe et al., 2008).

En el caso aquí reportado se realizó un examen de tomografía computarizada cone-beam que evidenció imagen compatible con hipertrofia bilateral del proceso coronoides mandibular.
El tratamiento de la hipertrofia del proceso coronoides mandibular, que se presenta como un problema mecánico, es principalmente quirúrgico (Wenghoefer et al.; Jamal et al.). Para la obtención de buenos resultados a largo plazo es fundamental también la kinesioterapia post-operatoria.

En conclusión, podemos afirmar que es importante recordar siempre que pacientes presentando apertura bucal limitada con evolución lenta, progresiva y asintomática deben pasar por el examen de procesos coronoides mandibulares. Podemos afirmar aún, que el examen de tomografía computarizada cone-beam tiene el potencial de ofrecer alta resolución en la imagen, presenta la ventaja de eliminar superposiciones auxiliando no solamente en el diagnóstico sino también en la planificación del procedimiento quirúrgico.

ALVES. N. \& FIGUEIREDO, D. N. Image of bilateral hypertrophy of the mandibular coronoid process through cone-beam computed tomography. Int. J. Odontostomat., 6(2):225-228, 2012.

ABSTRACT: The hypertrophy of the mandibular coronoid process is a rare condition, progressive and of unknown etiology, characterized by the over development of the coronoid process determining their size increased, resulting in progressive and painful limitation of mouth opening. The mandibular hypomobility is a present symptom in several diseases and associates it to a specific cause requires detailed clinical and imaginologic examination. The cone-beam computed tomography has the potential to provide complementary informations above than conventional radiography. It also has the advantage of eliminate overlaps, provide high resolution image, helping not only in diagnosis, but also in planning the surgical procedure. Here we report a case of male patient, who appeared at the clinic for an evaluation of a trismus. He had no pain, only discomfort for progressive limitation of mouth opening. On physical examination, only a limited mouth opening has been found. Although the movements of laterality and protrusión were posible, they were very limited. Cone-beam computed tomography examination was performed, that showed an image compatible with bilateral hypertrophy of the mandibular coronoid process.

KEY WORDS: hypertrophy, mandibular coronoid process, mandibular hypomobility, cone-beam computed tomography.

\section{REFERENCIAS BIBLIOGRÁFICAS}

Alves, N. \& Cândido, P. L. Anatomia para o cirurgiãodentista. São Paulo, Santos Editora, 2008.

Alves, N. \& Cândido, P. L. Anatomia para o curso de odontologia geral e específica. $3^{a}$ ed. São Paulo, Gen-Santos, 2012.

Iqbal, S.; Hamid, A. L. \& Purmal, K. Unilateral coronoid hyperplasia following trauma: a case report. Dent. Traumatol., 25(6):626-30, 2009.

Izumi, M.; Isobe, M.; Toyama, M.; Ariji, Y.; Gotoh, M.; Naitoh, M.; Kurita, K. \& Ariji, E. Computed tomographic features of bilateral coronoid process hyperplasia with special emphasis on patients without interference between the process and the zygomatic bone. Oral Surg. Oral Med. Oral Pathol. Oral Radiol. Endod., 99(1):93-100, 2005.

Jamal, B. T.; Taub, D. \& Gold, L. Contralateral coronoid hyperplasia in patients undergoing hemimandibulectomy with disarticulation: a case series. J. Oral Maxillofac. Surg., 67(9):1821-5, 2009.

Kai, S.; Hijiya, T.; Yamane, K. \& Higuchi Y. Open-mouth locking caused by unilateral elongated coronoid process: report of case. J. Oral Maxillofac. Surg., 55(11):1305-8, 1997. 
Mazzeto, M. O. \& Hotta, T. H. Hypertrophy of the mandibular coronoid process and structural alteration of the condyles associated with limited buccal opening: case report. Braz. Dent. J., 18(2):171-4, 2007.

Pregarz, M.; Fugazzola, C.; Consolo, U.; Andreis, I. A.; Beltramello, A. \& Gotte, P. Computed tomography and magnetic resonance imaging in the management of coronoid process hyperplasia: review of five cases. Dentomaxillofac. Radiol., 27(4):215-20, 1998.

Roychoudbury, A.; Gupta, Y. K.; Parkash, H. \& Karak, A. K. Jacob disease: report of a case and review of the literature. J. Oral Maxillofac. Surg., 60(6):699703, 2002.

Scarfe, W. C.; Farman, A. G. \& Sukovic P. Clinical applications of cone-beam computed tomography in dental practice. J. Can. Dent. Assoc., 72(1):7580, 2006.

Thiegui, R.; Galiè, M.; Piersanti, L. \& Clauser, L. Bilateral hiperplasia of the coronoid processes: clinical report. J. Craniofac. Surg., 16(4):723-6, 2005.

Wenghoefer, M.; Martini, M.; Allam, J. P.; Novak, N.; Reich, R. \& Bergé, S. J. Hyperplasia of the coronoid process in patients with ankylosing spondylitis (Bechterew Disease). J. Craniofac. Surg., 19(4):11148, 2008.
Dirección para correspondencia

Prof. Dr. Nilton Alves

Rua Humaitá, 1680

CEP: 14801-903.

Araraquara - SP

BRASIL

Email: niltonnalves@yahoo.com.br

Recibido : 27-02--2012

Aceptado: 12-07-2012 